\title{
Whole-Body Averaged Specific Absorption Rate Estimation using a Personal, Distributed Exposimeter
}

\author{
A. Thielens, P. Vanveerdeghem, S. Agneessens, Student Member, IEEE, P. Van Torre, G. Vermeeren, \\ H. Rogier, Senior Member, IEEE, L. Martens, Member, IEEE, and W. Joseph, Senior Member, IEEE
}

\begin{abstract}
For the first time, a body area network (BAN) is used to construct a personal, distributed exposimeter (PDE), which can measure the whole-body averaged specific absorption rate $\left(S_{A} A R_{w b}\right)$ in real life, together with the incident power density $\left(S_{\text {inc }}\right)$. The BAN consists of 4 textile antennas with integrated radio frequency receiver nodes tuned to the Global System for Mobile Communications 900 downlink band. Calibration measurements at $942.5 \mathrm{MHz}$, using a human subject, are performed in an anechoic chamber. These are combined with numerical simulations to estimate both $S A R_{w b}$ and $S_{\text {inc }}$ from the averaged received power on the PDE. The PDE has $50 \%$ prediction intervals of $3 \mathrm{~dB}$ on $S_{\text {inc }}$ and $3.3 \mathrm{~dB}$ on the $S A R_{w b}$, caused by the presence of the human body, whereas the best single textile antenna in our measurements exhibits $\mathrm{PI}_{50}$ 's of $7.1 \mathrm{~dB}$ on $S_{\text {inc }}$ and $5 \mathrm{~dB}$ on $\mathrm{SAR}_{\mathrm{wb}}$ Measurements using the PDE are carried out in Ghent (Belgium), during which a median $S_{\text {inc }}=47 \mu \mathrm{W} / \mathrm{m}^{2}$ and $S_{A} R_{\mathrm{wb}}=0.25 \mu \mathrm{W} / \mathrm{kg}$ are measured.
\end{abstract}

Index Terms-specific absorption rate, radio frequency, electromagnetic fields, exposure assessment, finite-difference time-domain simulations

\section{INTRODUCTION}

$\mathrm{T}$ HE absorption of radio frequency (RF) radiation is studied using the specific absorption rate (SAR). This quantity is defined as the ratio of the absorbed power over the mass in which this power is absorbed. The SAR is to be averaged over a certain volume or mass [1]. When considering far-field exposure, the most commonly studied SAR is the whole-body averaged SAR $\left(\mathrm{SAR}_{\mathrm{wb}}\right)$ [2]. However, this quantity can only be assessed using numerical simulations. Therefore, reference levels have been defined on the incident power density $\left(\mathrm{S}_{\text {inc }}\right)$ [1]. This $S_{\text {inc }}$ is usually measured using personal exposimeters (PEMs) [3-8]. These are body-worn devices, which contain a broadband antenna (typically recording between $0.1-6 \mathrm{GHz}$ [6-8]) and electronics to register the fields recorded by this antenna. The main advantage of these PEMs is that they

This paper is submitted for review on the 29th August 2014. This work was supported in part by the Flemish Fund for Scientific Research (FWO) under Grant No. 3G004612.

A. Thielens, P. Vanveerdeghem, S. Agneessens, P. Van Torre, G. Vermeeren, H. Rogier, L. Martens, and W. Joseph are with the Department of Information Technology, Ghent university/iMinds, Ghent, B-9050, Belgium (e-mail: arno.thielens@intec.ugent.be). register the $S_{\text {inc }}$ at the same time and location as the subject who is wearing the device. The main disadvantage of PEMs is that they record electric field strengths in proximity of the body instead of the $S_{\text {inc }}$ values for which reference levels are defined [1]. The $S_{\text {inc }}$ can be estimated from measurements using PEMs, but with a relatively large measurement uncertainty $[3,4-6,8]$. Consequently, the $\operatorname{SAR}_{w b}$, which can also be estimated using on-body measurements [7], will also exhibit a large measurement uncertainty. Recently, a novel technique using a body area network (BAN) of textile antennas was proposed [6]. Calibration measurements performed on this personal, distributed exposimeter (PDE) indicate that this device yields a lower measurement uncertainty on the $S_{\text {inc }}[6]$. However, the device has not been used for measurements in a real environment, nor has it been used to estimate the $\mathrm{SAR}_{\mathrm{wb}}$.

The goal of this paper is to calibrate a PDE for the Global System for Mobile Communications around $900 \mathrm{MHz}$ (GSM900) downlink (DL) band and use this PDE for measurements in a real environment. Simultaneously, numerical simulations are executed to determine the $\mathrm{SAR}_{\mathrm{wb}}$ in the same frequency band in different realistic environments. The calibration results and the numerical simulations can be combined to measure both the $S_{\text {inc }}$ and the $\mathrm{SAR}_{\mathrm{wb}}$ with a relatively low measurement uncertainty, using the PDE. To the authors' knowledge, this is the first time that such measurements are described.

\section{Materials AND MethodS}

A PDE is constructed, calibrated, and used for actual measurements of the $S_{\text {inc }}$ and $\mathrm{SAR}_{\mathrm{wb}}$ in the GSM $900 \mathrm{DL}$ band: $925-960 \mathrm{MHz}$. The PDE is a BAN composed of four textile antennas, designed for on-body usage, with integrated receiver electronics, tuned to the GSM 900 DL band. These are described in subsection $\mathrm{A}$. The on-body calibration of the antennas is described in subsection $\mathrm{B}$, while the numerical simulations to determine the $\mathrm{SAR}_{\mathrm{wb}}$ are described in subsection C. Subsection D describes how the calibration measurements and the numerical simulations can be combined to estimate the $\mathrm{SAR}_{\mathrm{wb}}$ from measurements with the PDE. The final subsection $E$ describes the measurements executed with the PDE in Ghent, Belgium. 


\section{A. Textile antennas and Receiver Electronics}

The PDE consists of four RF acquisition nodes. Every node is a combination of a textile antenna connected to receiver electronics tuned to the GSM 900 DL band, as shown in Fig. 1. The used textile antenna is a linearly polarized, quarter wavelength planar inverted-F antenna (PIFA) [6, 9]. The antennas have a size of $10 \times 12 \mathrm{~cm}^{2}$ and a mass smaller than $35 \mathrm{~g}$. The antenna is constructed entirely from lightweight and flexible materials, in order to maximize wearability. Furthermore, the conductive ground plane minimizes the influence of the body on the antenna performance [9]. The simulated on-body gain and $3 \mathrm{~dB}$ beam width are $2.9 \mathrm{~dB}$ and $110^{\circ}$ respectively, while the antenna efficiency is $76.6 \%$. The RF receiver electronics record the power $P_{r, i}$ received by textile antenna $i(i=1 . .4)$. They provide the geometrically averaged received power for every sample interval of $1 \mathrm{~s}$. This result is stored with a resolution of $1 \mathrm{~dB}$ and a sensitivity of $-72 \mathrm{dBm}$. The integrated receiver electronics are positioned on the antenna feed plane.

\section{B. Calibration Measurements}

The PDE is calibrated on the body of a subject in an anechoic chamber. The subject is a 26 years old male with a body mass index (BMI) of $22.6 \mathrm{~kg} / \mathrm{m}^{2}$. A calibration procedure is proposed to determine the effective on-body antenna aperture (AA) of a set of RF nodes placed on the subject's body and to select the positions of those nodes in order to minimize the measurement uncertainty. In this study, we have chosen to place the RF nodes on the torso of the subject, in order not to impede the subject's movements. The RF nodes are placed on one of 12 potential locations, A to L, on the front and back sections of the subject's torso. The linearly polarized RF nodes are placed either horizontally $(\mathrm{H})$, parallel to the subject's transverse plane or vertically $(\mathrm{V})$, orthogonal to the subject's transverse plane. The potential antennas' locations and polarizations are shown in Figure 1.

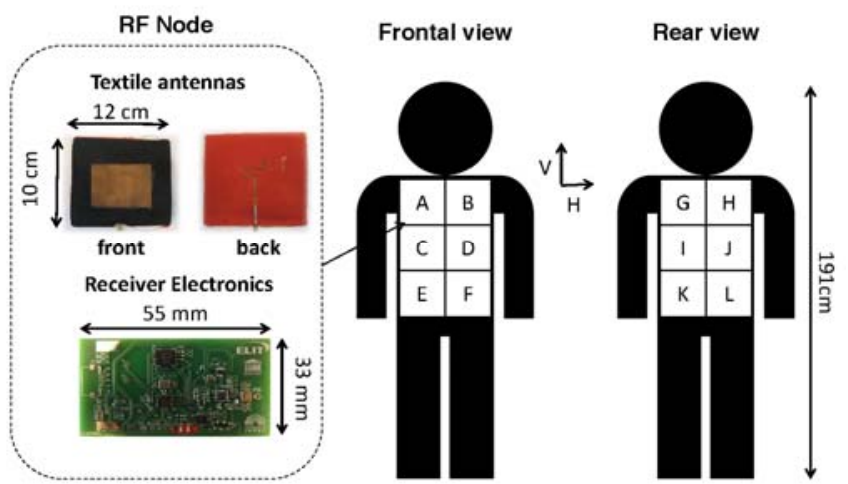

Fig. 1. Potential locations (A to $\mathrm{L}$ ) and polarizations ( $\mathrm{H}$ or $\mathrm{V}$ ) of the RF nodes on the subject's body.

First, the subject is rotated $360^{\circ}$ around his main axis in the far field of the transmitter (TX), a standard gain horn antenna emitting at $942.5 \mathrm{MHz}$ with an input power of $10 \mathrm{~mW}$. Each rotation is repeated for both $\mathrm{H}$ and $\mathrm{V}$ polarized TX. This results in 24 measurements of the on-body received power $P_{r, j}^{H}(\varphi)$ and $P_{r, j}^{V}(\varphi)(\mathrm{j}=\mathrm{A} . . \mathrm{L})$. Second, the free-space $S_{i n c}^{H}$ and
$S_{i n c}^{V}$ are measured using a NARDA broadband probe (NBM550 ), for both polarizations ( $\mathrm{H}$ and $\mathrm{V}$ ) of the TX. Third, $P_{r, j}^{H}(\varphi)$ and $P_{r, j}^{V}(\varphi)$ are geometrically averaged over combinations of 4 antennas. In this study, we only consider combinations of two V-polarized nodes and two H-polarized nodes, in order to reduce the polarization dependence of the PDE. Since there are 12 possible locations and 6 different ways to distribute two $\mathrm{V}$ polarized and two $\mathrm{H}$ polarized RF nodes over 4 selected positions, the total number of possible configurations is 2970 . These geometrically averaged received powers $P_{\text {geom }, l}^{H}(\varphi)$ and $P_{\text {geom,l }}^{V}(\varphi)$, with $l=1 . .2970$, can be used to calculate the geometrically averaged AA of each of the potential configurations of the PDE $\left(A A_{\text {geom }, l}\right)$ :

$$
A A_{\text {geom }, l}(\varphi, \psi)=\frac{P_{g \text { eoom }, l}^{H}(\varphi)}{S_{i n c}^{H}} \cos ^{2}(\psi)+\frac{P_{g e o m, l}^{V}(\varphi)}{S_{i n c}^{V}} \sin ^{2}(\psi)
$$

with $\psi$ the polarization of an incident electric field. In order to account for a realistic polarization, $A A_{\text {geom, },}(\varphi, \psi)$ is calculated for $10^{3} \psi$-samples, drawn from a Gaussian distribution for $\psi$ in an "Urban Macro cell" scenario [2,10], for each of 180 uniformly chosen $\varphi$ values in the interval $[0,2 \pi]$. This scenario is chosen because it corresponds best to the measurements that are carried out with the PDE in Ghent $[2,7]$. This results in a distribution of $A A_{\text {geom, }, l}$ in that scenario. For this distribution, the median value $p_{50}\left(A A_{\text {geom,l }}\right)$ and the $50 \%$ prediction interval $\left(\mathrm{PI}_{50,1}\right)$ of $\mathrm{AA}_{\text {geom,l }}$ are determined. This procedure is repeated 100 times for different $\psi$-samples, drawn from the same distribution, in order to ensure stability of the algorithm. The resulting $\mathrm{PI}_{50,1}$ is the ratio of the $75 \%$ and $25 \%$ percentile of the distribution and is directly proportional to the measurement uncertainty, due to the presence of the body during the measurements. Therefore, the configuration $l$ with the smallest $\mathrm{PI}_{50, \mathrm{l}}$ is chosen as the configuration used during measurements. The 4 RF nodes are then simultaneously deployed on the body at the chosen positions, according to the chosen polarizations, of configuration $l$, and are calibrated again using the same procedure. The $p_{50}\left(A A_{\text {geom, }, l}\right)$ and $\mathrm{PI}_{50, \mathrm{l}}$ are determined again and the distribution $\operatorname{Prob}\left(A A_{\text {geom }, l} \leq Z\right)$ is saved.

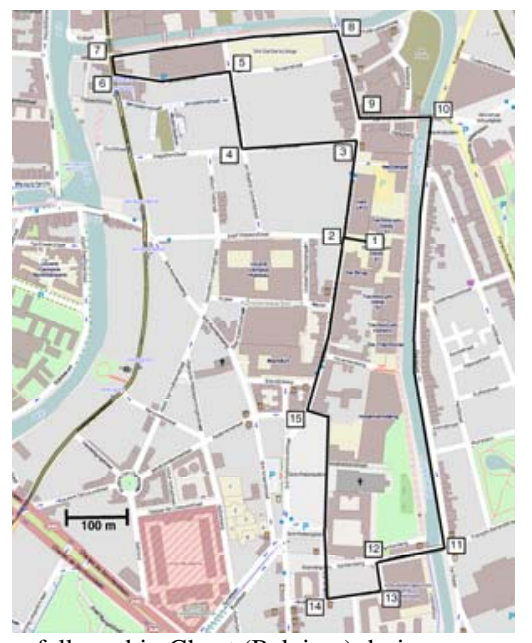

Fig. 2. Trajectory followed in Ghent (Belgium) during measurements (source: http://www.openstreetmap.org, 2014). 15 locations are indicated with white rectangles. 


\section{Numerical Simulations}

Finite-difference time-domain (FDTD) simulations are executed at $950 \mathrm{MHz}$ using the Virtual Family Male (VFM) [11]. The VFM is a heterogeneous phantom with a BMI equal to $22.3 \mathrm{~kg} / \mathrm{m}^{2}\left( \pm 0.5 \mathrm{~kg} / \mathrm{m}^{2}\right.$ compared to the subject’s BMI). The $\mathrm{SAR}_{\mathrm{wb}}$ for the VFM can be determined in the same 'Urban Macro cell' environment, using the method described in $[2,10]$. This method relies on the linearity of Maxwell's equations to determine the electric and magnetic fields on a closed surface around the VFM, by combining the fields obtained for single plane waves incident from different directions [2]. The $\mathrm{SAR}_{\mathrm{wb}}$ is calculated in 5000 multipath exposure samples in this environment, from which the distribution $\operatorname{Prob}\left(S A R_{w b} \leq Y \mid S_{\text {inc }}=1 \mathrm{~W} / \mathrm{m}^{2}\right)$ is determined [2].

\section{Estimating $S A R_{w b}$ from $P D E$ measurements}

From the calibration measurements, a distribution of the antenna aperture $\operatorname{Prob}\left(A A_{\text {geom, }} \leq Z\right)$, with $\mathrm{Z}$ a surface between 0 and $\infty \mathrm{m}^{2}$, is obtained, while from the numerical simulations and the method used in [2], a distribution of the $\mathrm{SAR}_{\mathrm{wb}}$ for a constant incident power density is determined. These two distributions can be combined to calculate the distribution of the $\mathrm{SAR}_{\mathrm{wb}}$ for a constant received power on the antennas, once the distribution of $A A_{\text {geom,l }}$ is inverted, using the definition of the antenna aperture:

$$
S_{\text {inc }}=\frac{P_{\text {geom }, l}}{A A_{\text {geom }, l}}
$$

For a constant $P_{\text {geom }}=1 W$, a distribution $\operatorname{Prob}\left(S_{\text {inc }} \leq\right.$ $\left.X \mid P_{\text {geom }}=1 \mathrm{~W}\right)$ can be obtained using (2). An expression for $\operatorname{Prob}\left(S A R_{w b} \leq Y \mid P_{\text {geom }}^{\text {meas }}=1 \mathrm{~W}\right)$ can be found using Bayes Theorem:

$\operatorname{Prob}\left(S A R_{w b} \leq Y \mid P_{\text {geom }}=1 \mathrm{~W}\right)=\int_{o}^{\infty} \operatorname{Prob}\left(S A R_{w b} \leq Y \mid S_{i n c}=\right.$ $X) \times \frac{d P r o b}{d X}\left(S_{\text {inc }} \leq X \mid P_{\text {geom }}=1 W\right) d X$

The $p_{50}\left(S A R_{w b} \mid P_{\text {geom }}=1 \mathrm{~W}\right)$ and $P I_{50}\left(S A R_{w b} \mid P_{\text {geom }}=\right.$ $1 W$ ) are determined for this distribution.

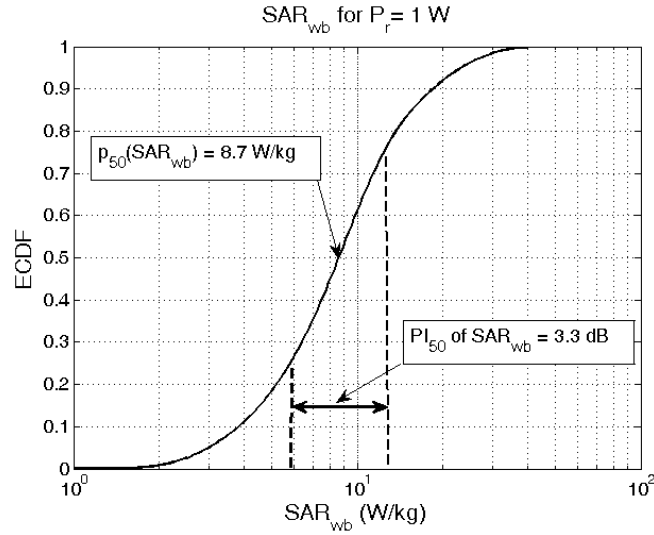

Fig. 3. Experimental cumulative distribution function (ECDF) of the $\mathrm{SAR}_{\mathrm{wb}}$ in the Urban Macro-cell scenario, for a constant received power of $1 \mathrm{~W}$ on the antenna.

\section{E. Measurements in Ghent Belgium}

Directly following the calibration measurements, the subject follows a 3.1-km-long outdoor trajectory through Ghent, Belgium, shown in Figure 2. During this walk, the powers received on the textile antennas are recorded and averaged over the 4 RF nodes. From these averaged powers, $S_{\text {inc }}$ and $S A R_{w b}$ values can be determined. These (median) values are then averaged over 6 minutes and compared to the ICNIRP RLs and BRs [1].

\section{RESULTS AND DISCUSSION}

In this section, first, the results of the calibration and numerical simulations are presented and discussed. Second, the results of our measurements performed in Ghent, using the PDE, are outlined.

\section{A. Calibration and Numerical Simulations}

A geometric averaging of the received power over $4 \mathrm{RF}$ nodes placed horizontally on position $B$, vertically on $D$,

TABLE I

PERFORMANCE CHARACTERISTICS OF THE PDE.

\begin{tabular}{cc}
\hline \hline Quantity & Value \\
\hline Frequency band & $\mathrm{GSM} 900 \mathrm{DL}$ \\
Selected positions and & $\mathrm{B}^{\mathrm{H}}, \mathrm{D}^{\mathrm{V}}, \mathrm{G}^{\mathrm{H}}, \mathrm{I}^{\mathrm{V}}$ \\
polarizations & $6.06 \pm 0.05$ \\
$p_{50}\left(A A_{\text {geom }}\right)\left(\mathrm{cm}^{2}\right)$ & $3.09 \pm 0.02$ \\
$\mathrm{PI}_{50}$ of $\mathrm{AA}$ geom $/ \mathrm{S}_{\text {inc }}(\mathrm{dB})$ & $0.104 \pm 0.001$ \\
$\mathrm{Detection} \mathrm{limit}\left(\mu \mathrm{W} / \mathrm{m}^{2}\right)$ & $8.7 \pm 0.5$ \\
$p_{50}\left(S A R_{w b} \mid P_{\text {geom }}=1 W\right)(\mathrm{W} / \mathrm{kg})$ & $3.3 \pm 0.6$ \\
$\mathrm{PI}_{50}$ of $\mathrm{SAR}_{\mathrm{wb}}(\mathrm{dB})$ & \\
\hline \hline
\end{tabular}

horizontally on G, and vertically on I (see Fig. 1), respectively, is found to lead to the lowest $\mathrm{PI}_{50}$. The isolation between the two antennas is larger than $27 \mathrm{~dB}$ averaged over the GSM 900 DL band. Table 1 shows the most important performance characteristics of the PDE after the calibration procedure. As Table 1 shows, the $\mathrm{PI}_{50}$ on $\mathrm{S}_{\text {inc }}$ equals $3.1 \mathrm{~dB}$. This value is a measure for the uncertainty caused by the human body and is low compared to the minimal and median values of $7.1 \mathrm{~dB}$ and $12 \mathrm{~dB}$, respectively, which are found for the single antennas, polarized either along $\mathrm{H}$ or $\mathrm{V}$ directions on positions $\mathrm{A}$ to $\mathrm{L}$. Previous studies using PEMs found values for the $\mathrm{PI}_{50}$ on the measured $\mathrm{S}_{\text {inc }}$ of $8.0 \mathrm{~dB}$ [5], using numerical simulations. For a PEM worn on the hips, $6.5 \mathrm{~dB}$ and $16 \mathrm{~dB}$, for $\mathrm{H}$ - and $\mathrm{V}$-polarized incident fields, respectively, are recorded [4]. A calibration of a couple of simultaneously worn PEMs on both hips of a subject showed a $\mathrm{PI}_{50}$ of 8.2 and $9.5 \mathrm{~dB}$ for the individual PEMs on the two hips and $4.8 \mathrm{~dB}$ for the combination of the two PEMs in a realistic environment [8]. For a previous prototype of the PDE using 3 RF nodes, we obtained a $\mathrm{PI}_{50}$ of $4.5 \mathrm{~dB}$ [6]. The $\mathrm{PI}_{50}$ listed in Table I is lower than all these values. This indicates that the PDE, presented here, can be used for measurements of $S_{\text {inc }}$ with less uncertainty.

Using the median $\mathrm{AA}_{\text {geom }}$ of $6.1 \mathrm{~cm}^{2}$ listed in Table 1, a detection limit of $0.104 \mu \mathrm{W} / \mathrm{m}^{2}$ can be obtained from the 
sensitivity of $-72 \mathrm{dBm}$. Commercial PEMs have a detection limit of $0.07 \mu \mathrm{W} / \mathrm{m}^{2}$, which is a factor of 1.5 lower than our detection limit. However, single PEMs tend to underestimate the incident power density by a factor larger than $1.5[3,8]$.

Figure 3 shows the distribution $\operatorname{Prob}\left(S A R_{w b} \leq Y \mid P_{\text {geom }}=\right.$ $1 W$ ) obtained using Eq. 3 for this configuration of RF nodes placed on the body. The $\mathrm{SAR}_{\mathrm{wb}}$ will be lower than $8.7 \mathrm{~W} / \mathrm{kg}$ in $50 \%$ of the cases, if a geometrically averaged power of $1 \mathrm{~W}$ is received on the antennas, see Fig. 3 and Table I. Note that a received power of $1 \mathrm{~W}$ on the antennas is relatively high, given the antenna aperture of $6 \mathrm{~cm}^{2}$. The $\mathrm{PI}_{50}$ on the $\mathrm{SAR}_{\mathrm{wb}}$ distribution of $3.3 \mathrm{~dB}$ is also relatively low compared to the minimal and median values of $5 \mathrm{~dB}$ and $10 \mathrm{~dB}$, respectively, found for the single antennas placed on positions A to L. However, it should be noted that there is an additional uncertainty (50\% prediction interval of $1.64 \mathrm{~dB}$ ) on the numerically obtained $\mathrm{SAR}_{\mathrm{wb}}$ values [12].

\section{B. Measurements in Ghent}

Figure 4 shows the $S_{i n c}$ (left vertical axis) and $S A R_{w b}$ (right vertical axis) values that are obtained by geometrically averaging the received powers over the 4 textile antennas with a sample rate of $1 \mathrm{~Hz}$ (full line) and averaged over 6 minutes (dashed). All measured data are above the detection limit. A median $S_{i n c}$ of $47 \mu \mathrm{W} / \mathrm{m}^{2}$ and a median $S A R_{w b}=0.25 \mu \mathrm{W} / \mathrm{kg}$ are measured along the full trajectory using the PDE. The maximally registered values are $S_{i n c}=4.9 \mathrm{~mW} / \mathrm{m}^{2}$ and $S A R_{w b}=26 \mu \mathrm{W} / \mathrm{kg}$. All the measured values, and thus also the values averaged over 6 minutes, are lower than the ICNIRP reference levels $\left(4.8 \mathrm{~W} / \mathrm{m}^{2}\right)$ and basic restrictions $(0.08 \mathrm{~W} / \mathrm{kg})$ for the general public. In [8], the incident power densities were measured in the same frequency band and area of Ghent. A $\mathrm{p}_{25}, \mathrm{p}_{50}, \mathrm{p}_{75}$, and $\mathrm{p}_{90}$ of $20,68,180$, and $340 \mu \mathrm{W} / \mathrm{m}^{2}$, respectively, were reported. During the measurements presented in Fig. 4, these values are 21, 47, 120, and 410 $\mu \mathrm{W} / \mathrm{m}^{2}$. These values are of the same order of magnitude. In [7] a $\mathrm{p}_{95}$ and $\mathrm{p}_{99}$ of 340 and $860 \mu \mathrm{W} / \mathrm{m}^{2}$ were reported in the same city. In this study these values are 0.83 and $2.2 \mathrm{~mW} / \mathrm{m}^{2}$. These values are higher, probably due to the sampling rate that is 10 times higher in this study than in [7], which inevitably increases percentiles higher than the $\mathrm{p}_{50}$.

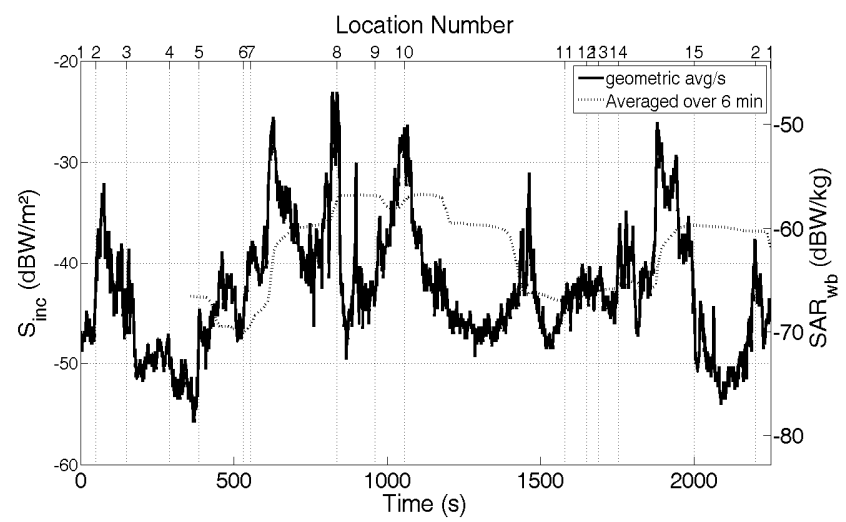

Fig. $4 S_{i n c}$ and $S A R_{w b}$ values measured along the trajectory. The location numbers on the upper horizontal axis correspond to those indicated in Fig. 2.

\section{CONCLUSIONS}

We have presented a calibration method of a particular body area network (BAN), denoted personal, distributed exposimeter (PDE), which measures the incident power density $\left(S_{i n c}\right)$ and, for the first time, real-life whole-body averaged specific absorption rate $\left(S A R_{w b}\right)$ in the Global System for Mobile Communications (GSM) 900 downlink (DL) band using 4 radio frequency (RF) power detection nodes integrated on 4 textile antennas. The PDE has a relatively low measurement uncertainty caused by the human body: $50 \%$ prediction intervals $\left(\mathrm{PI}_{50}\right)$ of $3.1 \mathrm{~dB}$ on $S_{\text {inc }}$ and $3.3 \mathrm{~dB}$ on $\mathrm{SAR}_{\mathrm{wb}}$ were measured for the PDE, whereas the best single textile antenna in our measurements exhibits $\mathrm{PI}_{50}$ of $7.1 \mathrm{~dB}$ on $S_{i n c}$ and $5 \mathrm{~dB}$ on $\mathrm{SAR}_{\mathrm{wb}}$. The measurement uncertainty is also low compared to existing solutions in literature. The PDE is used for real measurements in Ghent (Belgium) where a median $S_{i n c}$ of $47 \mu \mathrm{W} / \mathrm{m}^{2}$ and $S A R_{w b}=$ $0.25 \mu \mathrm{W} / \mathrm{kg}$ were measured.

\section{REFERENCES}

[1] International Commission on Non-Ionizing Radiation Protection. 1998. Guidelines for limiting exposure to time-varying electric, magnetic, and electromagnetic fields (up to $300 \mathrm{GHz}$ ). Health Physics 74, pp. 494-522.

[2] Vermeeren G, Joseph W, Olivier C, Martens L. 2008. Statistical multipath exposure of a human in a realistic electromagnetic environment. Health Physics 94: pp. 345 - 54.

[3] Iskra S, McKenzie R, Cosic I. 2010. Factors influencing uncertainty in measurement of electric fields close to the body in personal RF dosimetry. Radiat Prot Dosimetry 140 (1), pp. 25-33.

[4] Bolte J.F.B., Van der Zande G., Kamer J., 2011. Calibration and uncertainties in personal exposure measurements of radiofrequency electromagnetic fields. Bioelectromagnetics 32(8), pp. 652-663.

[5] Neubauer G, Cecil S, Giczi W, Petric B, Preiner P, Fröhlich J, Röösli M. 2010. The association between exposure determined by radiofrequency personal exposimeters and human exposure: a simulation study. Bioelectromagnetics 31, pp. 535-545.

[6] Thielens A, De Clerq H, Agneessens S, Lecoutere J, Verloock L, Declerq F, Vermeeren G, Tanghe E, Rogier H, Puers R, Martens L, Joseph W. 2013. Distributed on Person Exposimeters for Radio Frequency Exposure Assessment in Real Environments. Bioelectromagnetics 34 (7), pp. 563-567.

[7] Joseph W, Vermeeren G, Verloock L, Heredia MM, Martens L. 2008.Characterization of Personal RF Electromagnetic Field Exposure and Actual Absorption for the General Public. Health Physics 95(3), pp. 317-330.

[8] Thielens A, Angeessens S, Verloock L, Tanghe E, Rogier H, Martens L, Joseph W. 2014. On-Body Calibration and Processing for a Combination of Two Radio Frequency Personal Exposimeters. Radiat. Prot. Dosi., doi:10.1093/rpd/ncu056

[9] Hertleer C, Tronquo A, Rogier H, Vallozzi L, Van Langenhove L. 2007. Aperture-Coupled Patch Antenna for Integration Into Wearable Textile Systems. IEEE Antennas and Wireless Propagation Letters 6, pp.392395.

[10] Thielens A, Vermeeren G, Joseph W, and Martens L. 2013. Stochastic Method for Determination of the Organ-Specific Averaged SAR in Realistic Environments at $950 \mathrm{MHz}$. Bioelectromagnetics 34(7): 549562.

[11] Christ A, Kainz W, Hahn EG, Honegger K, Zefferer M, Neufeld E, Rascher W, Janka R, Bautz W, Chen J,Kiefer B, Schmitt P, Hollenbach HP, Shen J, Oberle M, Szczerba D, Kam A, Guag JW, and Kuster N. 2010. The Virtual Family - development of surface-based anatomical models of two adults and two children for dosimetric simulations. Phys Med Biol 48:N23-N38.

[12] Bakker JF, Paulides MM, Christ A, Kuster N, van Rhoon GC. 2010. Assessment of induced SAR in children exposed to electromagnetic plane waves between $10 \mathrm{MHz}$ and $5.6 \mathrm{GHz}$. Phys Med Biol 55(11): 3115-3130. 